\title{
Umbilical Cord Mesenchymal Stem Cell Therapy for Regenerative Treatment of Rheumatoid Arthritis: Opportunities and Challenges
}

\author{
Xiaolan $L v^{1, *}$ \\ Liming Wang ${ }^{2, *}$ \\ XiaoRong Zou ${ }^{3}$ \\ Shigao Huang $\mathbb{D}^{4}$ \\ 'Department of Laboratory Medicine, \\ Liuzhou Maternity and Child Healthcare \\ Hospital, Liu Zhou, Guang Xi, People's \\ Republic of China; ${ }^{2}$ Shaanxi Jiuzhou \\ Biomedical Science and Technology \\ Group, Xi'an, Shaan Xi, People's Republic \\ of China; ${ }^{3}$ Department of Hematology, \\ 986 Hospital of Fourth Military Medical \\ University, Xi'an, Shaan Xi, People's \\ Republic of China; ${ }^{4}$ Faculty of Health \\ Sciences, University of Macau, Macau, \\ People's Republic of China
}

*These authors contributed equally to this work
Correspondence: Shigao Huang Email huangshigao2010@aliyun.com

\begin{abstract}
Rheumatoid arthritis (RA) is an autoimmune disease of unknown etiology with a high rate of disability. Traditional treatments for RA remain a challenging issue. For example, nonsteroidal anti-inflammatory drugs (NSAIDs) have no therapeutic effects on joint destruction, and the prominent side effects include gastrointestinal symptoms. RA is characterized by recurrence and bone attrition. Therefore, regenerative medicine and the use of umbilical cord mesenchymal stem cell (UC-MSC) therapies have recently emerged as potential options. UC-MSCs are multifunctional stem cells that are present in neonatal umbilical cord tissue and can differentiate into many kinds of cells, which have broad clinical application prospects in the tissue engineering of bone, cartilage, muscle, tendon, ligament, nerve, liver, endothelium, and myocardium. Moreover, UC-MSCs have advantages, such as convenient collection of materials and no ethical disputes; thus, these cells have attracted increasing attention from researchers. However, there are few clinical studies regarding UC-MSC therapy for RA. In this paper, we will review traditional drugs for RA treatment and then focus on UC-MSC therapy for RA, including preclinical and clinical UCMSC applications for RA patients in the context of regenerative medicine. Finally, we will summarize the challenges and perspectives of UC-MSCs as a potential therapeutic strategy for RA. This review will help to design and discover more potent and efficacious treatments for RA patients and aid in advancing this class of cell therapy.
\end{abstract}

Keywords: cell-based therapies, rheumatoid arthritis, mesenchymal stem cells, umbilical cord, regenerative medicine

\section{Introduction}

Rheumatoid arthritis (RA) is a systemic, inflammatory autoimmune disease that mainly involves peripheral facet joint disease, ${ }^{1}$ which is the main pathological feature, as well as joint synovial cell proliferation, inflammatory cell infiltration, and pannus. ${ }^{2}$ To date, traditional RA treatment mainly involves reducing the symptomatic inflammatory reaction and sequelae ${ }^{3}$ conventional treatment cannot satisfy the clinical requirement of achieving a curative effect. ${ }^{4}$ Therefore, exploring more effective and safer treatment options is increasingly important. Mesenchymal stem cells (MSCs) are derived from early-development mesoderm pluripotent stem cells and have a high degree of self-renewal and multidirectional differentiation potential; MSCs widely exist in a variety of human tissues and can be cultured in vitro. Under specific conditions, MSCs can differentiate into osteoblasts, nerve cells, adipose cells, muscle cells, and cardiomyocytes. These cells have great 


\section{Graphical Abstract}

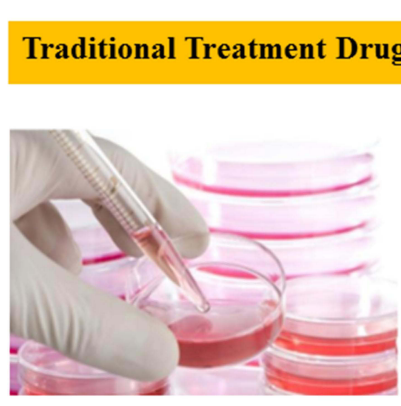

Preclinical experiment

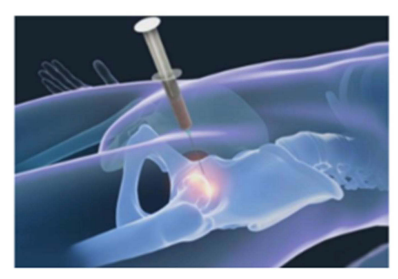

Clinical Study
No satisfactory effect

Advantages of UC-MSCs Therapy for RA application value in tissue engineering repair and in cell replacement therapy. ${ }^{5}$ First, MSCs can differentiate into bone and cartilage. Second, these cells also inhibit immunity, alleviate inflammation, resist fibrosis, and promote vascular repair. Umbilical cord MSCs (UC-MSCs) are derived from human umbilical cord blood and have MSC characteristics and functions. Therefore, UC-MSCs offer the hope of a new treatment option for RA. In recent years, the use of UC-MSCs to treat rheumatic immune diseases has garnered increasing attention, ${ }^{6-9}$ and the promising results of preclinical studies suggest that UC-MSCs may be a therapeutic method for regenerative medicine. ${ }^{7,10,11}$ However, there have been few studies regarding the effects of UC-MSCs on patients with RA who had recurrent symptoms after long-term treatment with regular agents in the clinic. However, there is still a pressing need for examining efficiency and safety considerations of UCMSCs in clinical investigations. This review aims to summarize the current preclinical experimental and clinical studies of RA treatment with UC-MSCs, examining the opportunities and challenges in research development progress.

Traditional Treatment Drugs for RA According to the development time and principles, the current drugs for treating RA are divided into four generations: the first generation is nonsteroidal anti-inflammatory drugs (NSAIDs); the second generation is glucocorticoids; the third generation is disease-changing drugs (slow-acting antirheumatic drugs, SAARDs); and the fourth generation are early biologic agents dominated by tumor necrosis factor (TNF)- $\alpha$ inhibitors. The following are the descriptions of the difficulties of these four drug types in RA treatment.

First, NSAIDs ${ }^{12}$ are the first-generation treatment for RA and represent drugs such as aspirin and diclofenac. These drugs inactivate cyclooxygenase (COX)-1 and COX-2 mainly through acetylation. ${ }^{13}$ NSAIDs have no therapeutic effect on joint destruction, and the prominent side effects are gastrointestinal symptoms. In recent years, newly marketed specific COX-2 inhibitors, such as meloxicam and celecoxib, have not affected COX-L due to their specific inhibition of COX-2. ${ }^{14}$ Thus, this treatment can avoid the traditional gastrointestinal side effects of NSAIDs. Second, disease-modifying antirheumatic drugs (DMARDs) $^{15}$ or SAARDs are third-generation therapies for RA. This class of drugs includes antimalarial drugs, gold preparations, and cytotoxic drugs. DMARDs mainly control the development of joint disease by reducing synovial inflammation and even play a role in repair, but most of these agents have serious side effects, such as vomiting, skin rash, leukocyte reductions, and liver and kidney function damage. Third, glucocorticoids are the secondgeneration treatment for RA. ${ }^{16}$ The mechanism of action 
is that glucocorticoids bind to glucocorticoid receptors and reach the nucleus, lowering the activity of nuclear factor kappa-light-chain-enhancer of activated B cells (NF- $\kappa \mathrm{B}$ ) and reducing the production of proinflammatory cytokines to effectively reduce inflammation. These drugs cannot block the progression of RA or joint destruction, and longterm use can induce side effects such as infection, cortical hyperfunction, osteoporosis and hypertension. However, small doses and short courses of treatment can reduce symptoms through anti-inflammatory and anticleric effects.

Finally, early biological agents, mainly TNF- $\alpha$ inhibitors, are the fourth generation of drugs for the treatment of RA. ${ }^{17}$ Because of the advantages of high pharmacological selectivity and low toxicity and side effects, biologics will have wide application prospects. Among the biologics used for the treatment of RA, the most clinically studied are TNF- $\alpha$ inhibitors. Three TNF- $\alpha$ inhibitors, etanercept, infliximab and adalimumab, are currently approved for the treatment of RA. ${ }^{18}$ The most common adverse reactions are injection-site reactions and infection. Infliximab, developed by Centocor, is used for the treatment of patients with early RA, ${ }^{19}$ and the common adverse reactions are infection and allergic reactions in some patients. ${ }^{20}$ Adalimumab, developed by Abbott, is the first human TNF antagonist and has adverse reactions such as nasopharyngitis and upper respiratory tract infection, and patients are prone to tuberculosis recurrence. ${ }^{14,21}$ In addition, compared with the use of methotrexate (MTX; $30.9 \%$ ), the risk of opportunistic infections and invasive melanoma increased by $50 \%$ in patients treated with TNF antagonists $(40.1 \%)$ compared with those treated with TNF antagonists. ${ }^{22}$ These findings limit the wide application of biological agents to some extent.

\section{Advantages of UC-MSC Therapy for RA}

MSCs are multipotent cells derived from the heart, peripheral blood, umbilical cord blood, muscle, lung, trabecular bone, intestinal tract, kidney, liver, pancreas, synovium, skin, and even the brain and can be a promising form of regenerative medicine. ${ }^{23}$ In recent years, MSCs have been widely used in the treatment of various diseases. ${ }^{24}$ In China, MSCs have been used to treat some clinically refractory diseases, such as spinal cord injury, cerebral palsy, amyotrophic lateral sclerosis, systemic lupus erythematosus, systemic sclerosis, Crohn's disease, stroke, diabetes, diabetic foot, and cirrhosis, among other conditions. ${ }^{25-27}$ Clinical practice shows that MSCs are effective treatments for many diseases, such cotransplantation with hematopoietic stem cells, the enhancement of hematopoietic function, and implantation with hematopoietic stem cell grafts to treat graft-versus-host disease (GVHD). ${ }^{24}$ In clinical research, the main types of MSCs are bone marrow-derived MSCs (BM-MSCs), adipose-derived mesenchymal stromal cells (AD-MSCs) and UC-MSCs.

RA is an inexorably progressive lung disease of unknown origin. ${ }^{28,29}$ The prognosis is poor, limited treatment options are available, and the median survival remains just 3-5 years. ${ }^{30}$ Despite the use of pirfenidone and nintedanib for the treatment of RA, MSCs have potential as therapies for RA. ${ }^{3}$ In recent years, based on the biological characteristics of MSCs, much research has been done on the role of MSCs in autoimmune diseases. ${ }^{31}$ In particular, MSCs can reduce the incidence of such diseases, especially RA, can also reduce inflammation after disease onset and can delay further disease development. ${ }^{32}$ MSCs have immunoregulatory roles, regulating the expression of cytokines by $\mathrm{T}$ cells, B cells, dendritic cells (DCs), and natural killer (NK) cells. ${ }^{33,34}$ However, RA is mainly caused by autoimmune disease mediated by $\mathrm{T}$ cells and involves B cells, cytokines, apoptosis, proteases, and other factors. ${ }^{35}$ Therefore, the use of MSCs to intervene in the pathological basis of RAmediated inflammation has become a feasible method. Nearly 100 studies have been conducted to establish experimental models for MSC treatment of RA. In most experimental models, the efficacy of MSC treatment was demonstrated by significantly reducing the induction and progression of experimental arthritis. The most widely used model is the collagen-induced arthritis (CIA) model. ${ }^{36}$ These promising models pave the way for MSC treatment of human RA disease. Currently, nine clinical trials are active, and as a consequence, the clinical data are not publicly available, although the MSCs are known to have included 50\% UC-MSCs, 25\% AD-MSCs, and 25\% BM-MSCs. ${ }^{9}$

Compared with BM-MSCs and AD-MSCs, UC-MSCs have the following advantages: cell surface expression of major histocompatibility complex I (MHC I) is lower, and MHCII is not expressed, so UC-MSCs seldom cause immune rejection. Additionally, the growth environment of UC-MSCs more pure than that of other MSCs, these cells have stronger abilities to proliferate and differentiate and are easy to culture in vitro. Furthermore, UC-MSCs 
are conveniently derived from umbilical cords, so there are fewer ethical issues, and they do not easily transmit disease. ${ }^{37}$

\section{Preclinical Experiments}

\section{Regulation of the Immune Response}

UC-MSCs mediate the differentiation, proliferation, and activation of $\mathrm{T}$ cell subsets in various ways and inhibit B cell proliferation and differentiation, as well as the maturation of DCs and NK cell activity to achieve various immunomodulatory effects. ${ }^{6}$ Therefore, UC-MSCs may be a feasible method to intervene in the pathological basis of RA-associated inflammation. Recent studies on the treatment of RA suggest that UC-MSCs suppress the various inflammatory effects of fibroblast-like synoviocytes (FLSs) and T cells in vitro and attenuate the development of CIA in vivo. ${ }^{38}$ MSCs have low immunogenicity and express MHC-I molecules but not MHC-II molecules and costimulatory molecules such as B7-1, B7-2, CD40, CD40, and Fas ligand. MSCs are not restricted by MHC molecules, so they play an immunomodulatory role in vivo. ${ }^{39}$ Researchers ${ }^{40}$ have demonstrated that UC-MSCs have an immunomodulatory effect on allogeneic $\mathrm{T}$ lymphocytes and can inhibit the proliferation and changes in $\mathrm{T}$ lymphocytes stimulated by other cell subsets and cytokines secreted by $\mathrm{T}$ cells in the context of immune responses. The effects of UC-MSCs on the immune regulation of Th17 cells in RA patients have been reported by Wang et al. ${ }^{41}$ The results showed that UC-MSCs could significantly reduce the numbers of Th17 cells and the expression of cytokines and reduce the expression of ROR- $\gamma \mathrm{t}$ and serum levels IL-17, and there was a good correlation with the dose. UC-MSCs were observed in vitro in a mixed cell culture with peripheral blood mononuclear cells (PBMCs) from RA patients. In vitro, the Th17 level was downregulated in the highly active group, but no similar regulation was observed in the normal and mildly active groups, suggesting that the immunoregulatory effect of UC-MSCs on Th17 cells may be regulated by other factors. Gu et $\mathrm{al}^{42}$ concluded that UCMSCs could increase the peripheral blood Treg proportion in CIA rats and inhibit secretion by Th17 cells and the activity of neutrophils. UC-MSCs can also decrease the release of proinflammatory factors and induce immune reconstruction, in addition to reducing the immune inflammatory reaction. Therefore, UC-MSCs may provide a new way to treat RA. Another intriguing feature of MSCs is that they can escape immune recognition and inhibit immune responses and could become a potential tool for immunomodulatory cell therapy in immune-mediated diseases. $^{43}$

\section{Injury Repair}

UC-MSCs have strong proliferative abilities and multidirectional properties and can differentiate into adult cells in vivo or in vitro under appropriate environments. ${ }^{44} \mathrm{UC}$ MSCs have the capacity for tissue repair and regeneration. UC-MSCs secrete a range of growth factors, such as hepatocyte growth factor (HGF), vascular endothelial growth factor (VEGF), stromal cell-derived factor-1 (SDF-1), keratinocyte growth factor (KGF), fibroblast growth factor (FGF), and insulin-like growth factor-1 (IGF-1), to facilitate proliferation and tissue damage repair. ${ }^{45,46}$ MSCs have the potential to be pluripotent and capable of giving rise to a variety of cell types. The properties of various factors, growth factors, and extracellular vesicles can be transmitted. ${ }^{47} \mathrm{NF}-\mathrm{\kappa B}$ and other cellular signaling pathways can be affected to treat diseases. ${ }^{48}$ After intravenous injection, UC-MSCs can differentiate into alveolar epithelial cells and pulmonary vascular endothelial cells. ${ }^{49}$ Additionally, UC-MSCs can play a role in injury repair. There is evidence that angiopoietin-1 (ANG-1) and angiopoietin-2 (ANG-2) are secreted with KGF to repair damage. MSCs have homing abilities, can migrate to injured sites, and can differentiate into local components of injured sites and help tissue regeneration by secreting chemokines, cytokines, and growth factors ${ }^{45}$. Hence, UC-MSCs can be widely used in articular cartilage repair.

\section{Reduction in the Inflammatory Response}

UC-MSCs play a role in inflammation at the site of injury to mitigate the inflammatory response. Active migration to the injured site for repair is called the "return" of the MSCs' "nest function," and a strong tendency has been demonstrated in animal experiments in vivo under various microenvironmental conditions. ${ }^{50,51}$ Researchers have explored how the immunomodulatory effects of nodules are mediated by UC-MSCs in vitro. ${ }^{52-55}$ UC-MSCs can express a variety of MSC markers and a variety of stem cell-related genes, are cells with the potential for multidirectional differentiation, and have low immunogenicity, which may be due to the low level of MHC-I antigens and no expression of MHC-II antigens or FasL. The effects of these cells are not related to the expression of 
costimulatory molecules associated with $\mathrm{T}$ cell activation. ${ }^{56}$ UC-MSCs significantly reduce inflammation by extensively downregulating inflammatory products or cytotoxic mediators that act on immune cells and increase the level of the anti-inflammatory cytokine IL-10. ${ }^{57}$ In addition to suppressing the immune response, UC-MSCs induce the production of IL-10-secreting regulatory $\mathrm{T}$ cells (Tregs) during local inflammation. Il-10, a characteristic Treg cytokine, plays an important role in controlling autoantigen-responsive $\mathrm{T}$ cells in vivo, and these Tregs inhibit the effects of effector $\mathrm{T}$ cells on antigens. UC-MSCs extensively reduce cytokines and chemokines and directly activate macrophages. ${ }^{58}$

Greish et $\mathrm{al}^{59}$ used UC-MSCs to treat an RA rat model induced with complete Freund's adjuvant (CFA), and the results showed that the group of rats that received hematopoietic stem cells (HSCs) and the group of rats that received UC-MSCs both showed improvements in arthritis symptoms after 21 days of treatment, and arthritis symptoms completely disappeared after 34 days. The mean diameter in the UC-MSC group was only half that of the control group and of the MTX group, and that of the UCMSC group was $0.6 \mathrm{~mm}$ smaller than that of the HSC group. Serum levels of TNF- $\alpha$, IFN- $\gamma$, and IL- 1 in the UCMSC group were significantly decreased compared with those of the control group and the MTX group, while the expression of IL-10 was increased. Hematoxylin-eosin (HE) staining showed that UC-MSCs could significantly reduce leukocyte infiltration and synovial tissue hyperplasia in the articular cavity. Masson staining showed edema in the intrasynovial vessels in almost all arthritic rats, with vacuoles forming in the vessel walls, ultimately leading to hemorrhagic necrosis. Additionally, the UC-MSC group also showed a reduction in extensive fibrosis of the joint space, which verified that UC-MSCs could improve the symptoms of CFA-induced RA, and the mechanism of action may be through regulating the expression of cytokines and improving pathological changes.

\section{Clinical Research}

At present, many universities, medical institutions, scientific research institutions, and enterprises have launched dozens of MSC treatments for RA disease. Related clinical studies are proceeding. By July 2020, according to ClinicalTrials.gov, an international clinical trial registry, multiple clinical trials of UC-MSCs for the treatment of RA have been recorded. There have been as many as six application cases, which have been organized by domestic units and Korean affiliates, using UC-MSCs either alone or combined with DMARDs. The interventions, outcomes, measures, and study designs were analyzed. Information on the clinical trial Phase Is shown in Table 1. Domestic research is mostly included. There was one clinical trial abroad with applicants from Korea (NCT03618784). The study designs were similar to the domestic studies, and the evaluation included erythrocyte sedimentation rate (ESR), Korean Health Assessment Questionnaire (KHAQ), clinical disease activity index (CDAI), $100 \mathrm{~mm}$ pain VAS and changes in cytokines.

In most clinical studies, UC-MSCs extensively reduced cytokines and chemokines and directly activated macrophages. ${ }^{8}$ The pathogenesis of RA and its immune mechanisms are related to cytokine disorders. UC-MSCs are widely available, and their immune rejection is low. Based on preclinical studies, UC-MSCs have very good immune regulation and are able to repair tissue damage. Therefore, the clinical application of cell therapy for RA has achieved a certain effect. Many National Clinical Trials (NCTs) registered on ClinicalTrials.gov are shown in Table 1. Most of the studies are from China, and only two FURESTEM-RA Inj trials are from Korea, the latter of which evaluated general indices such as ACR20/50/70, EULAR, DAS28-ESR, and KHAQ and detected changes in cytokines (TNF- $\alpha$, IL-1 $\beta$, IL-4, IL-6, IL-8, IL-10, IL-13, IL-17A, IL-21, and IL-22). ${ }^{60}$ In South Korea, a multicenter randomized double-blind parallel placebocontrolled phase I/IIa RA clinical trial (NCT03618784) administered allogeneic UC-MSCs (named FURESTEMRA Inj) to 33 RA patients. Clinical and safety parameters were monitored for one month following the infusion of UC-MSCs. No serious adverse events or major abnormalities in serum chemical or hematologic profiles were observed. Early clinical studies of the use of UC-MSCs in RA patients have shown promising safety profiles and could significantly improve symptoms and repair damaged tissue. ${ }^{7,61}$ Phase 1 clinical trials have been conducted to evaluate the safety of UC-MSC therapy.

Our previous study was registered at ClinicalTrials.gov (NCT01547091), ${ }^{8}$ in which UC-MSCs were intravenously injected into RA patients. A total of 172 patients were divided into two groups: those who received DMARDs or those who received DMARDs plus UC-MSCs $\left(4 \times 10^{7}\right.$ cells per treatment). Diet, sleep, physical strength, fatigue, and other clinical symptoms were examined, and significant improvements were observed. For the UC-MSC treatment group, all patients met the ACR20 standard. Twelve 
Table I Clinical Trial of Umbilical Cord Mesenchymal Stem Cells Therapy of RA from ClinicalTrials.gov

\begin{tabular}{|c|c|c|c|c|c|c|}
\hline $\begin{array}{l}\text { NCT } \\
\text { Number }\end{array}$ & Interventions & Outcome Measures & Age & Phases & Enrollment & Study Designs \\
\hline NCT02643823 & $\begin{array}{l}\text { hUC-MSC } \\
\text { +DMARDs; } \\
\text { DMARDs }\end{array}$ & SAE; RA Serology; DAS 28 Index & $18-80$ & Phase I & 40 & $\begin{array}{l}\text { Allocation: Randomized } \\
\text { Intervention Model: Parallel } \\
\text { Assignment } \\
\text { Masking: None (Open Label) } \\
\text { Primary Purpose: Treatment }\end{array}$ \\
\hline NCT0I985464 & hUC-MSC & $\begin{array}{l}\text { AE;28-DAS; EULAR; QOL; HAQ; } \\
\text { at months } 3 \text { and I } 2 \text { of CRP; ESR } \\
\text { anti-CAM; RF; EULAR }\end{array}$ & $\geq 18$ & $\begin{array}{l}\text { Phase II } \\
\text { Phase } 2\end{array}$ & 20 & $\begin{array}{l}\text { Allocation: N/A } \\
\text { Intervention Model: Single Group } \\
\text { Assignment } \\
\text { Masking: None (Open Label) } \\
\text { Primary Purpose: Treatment }\end{array}$ \\
\hline NCT0I54709I & $\begin{array}{l}\text { UC-MSCs; } \\
\text { DMARDs; } \\
\text { UCMSC } \\
\text { +DMARDs }\end{array}$ & $\begin{array}{l}\text { Safety of MSC treatment; RA } \\
\text { Serology; DAS } 28 \text { Index; Patient's } \\
\text { assessment of pain. }\end{array}$ & $18-70$ & $\begin{array}{l}\text { Phase II } \\
\text { Phase } 2\end{array}$ & 200 & $\begin{array}{l}\text { Allocation: Non-Randomized } \\
\text { Intervention Model: Single Group } \\
\text { Assignment } \\
\text { Masking: None (Open Label) } \\
\text { Primary Purpose: Treatment }\end{array}$ \\
\hline NCT03828344 & $\begin{array}{l}\text { hUC-MSC; } \\
\text { Placebo }\end{array}$ & $\begin{array}{l}\text { Frequency of AE; SAE } \\
\text { All following indexes at Week } 12 \\
\text { and Week 24:ACR20; ACR50; } \\
\text { ACR70; DAS28-CRP; HAQ-DI } \\
\text { score; SDAI; RF; anti-CCP }\end{array}$ & $18-70$ & Phase I & 16 & $\begin{array}{l}\text { Allocation: Randomized } \\
\text { Intervention Model: Parallel } \\
\text { Assignment } \\
\text { Masking: Quadruple (Participant, } \\
\text { Care Provider, Investigator, } \\
\text { Outcomes Assessor) } \\
\text { Primary Purpose: Treatment }\end{array}$ \\
\hline NCT03798028 & UC-MSCs & $\begin{array}{l}\text { BT; HGB; FVC; DLCO; ACR 20; } \\
\text { ACR 50; ACR 70; WBC; PC; FVC } \\
\text { DLCO; Lung CT; 6MWT }\end{array}$ & $18-70$ & $\begin{array}{l}\text { Not } \\
\text { Applicable }\end{array}$ & 250 & $\begin{array}{l}\text { Allocation: Randomized } \\
\text { Intervention Model: Parallel } \\
\text { Assignment } \\
\text { Masking: Single (Participant) } \\
\text { Primary Purpose: Treatment }\end{array}$ \\
\hline NCT03618784 & $\begin{array}{l}\text { FURESTEM-RA } \\
\text { Inj; Sterile } \\
\text { saline }\end{array}$ & $\begin{array}{l}\text { AE; ACR20,50,70; EULAR; } \\
\text { DAS28-ESR; KHAQ; CDAl; } \\
\text { I00mm Pain VAS; consumed } \\
\text { amount of rescue medicine; } \\
\text { Change in Cytokine (TNF-a, IL- } \\
\text { Ib, IL-4, IL-6, IL-8, IL-10, IL-I3, } \\
\text { IL-I7A, IL-2I, IL-22) }\end{array}$ & $19-80$ & $\begin{array}{l}\text { Phase II } \\
\text { Phase } 2\end{array}$ & 33 & $\begin{array}{l}\text { Allocation: Randomized } \\
\text { Intervention Model: Parallel } \\
\text { Assignment } \\
\text { Masking: Quadruple (Participant, } \\
\text { Care Provider, Investigator, } \\
\text { Outcomes Assessor) } \\
\text { Primary Purpose: Treatment }\end{array}$ \\
\hline
\end{tabular}

Notes: Placebo, same suspension as BX-U00I but without cells. NCT Number was identified by ClinicalTrials.gov Identifier in Medline.

Abbreviations: UC-MSCs, umbilical cord derived mesenchymal stem cells; DAS 28, Disease Activity Score 28 joint; AE, adverse events; ESR, erythrocyte sedimentation rate; RF rheumatoid factor; DMARDs, drug rheumatoid arthritis with disease-modifying drugs; SAE, serious adverse events; DAS28-CRP, disease activity score 28-joint count using $\mathrm{C}$ reactive protein; HAQ-DI, health assessment questionnaire disability index; SDAI, Simplified Disease Activity Index; anti-CCP, anti-cyclic citrullinated peptide; HGB, hemoglobin; FVC, forced vital capacity; ACR, American College of Rheumatology; EULAR, European League Against Rheumatism; KHAQ, Korean Health Assessment Questionnaire; CDAl, clinical disease activity index; VAS, visual analogue scale; QOL, quality of life measure; CRC, reactive protein; anti-CAM, anti-citrulline antibody measure; BT, blood routine; DLCO, diffusing lung capacity for carbon monoxide; 6MWT, six-minute walk test; WBC, white blood cell count; PC, platelet count; IL, interleukin; FURESTEM-RA Inj, the allogeneic umbilical cord blood-derived mesenchymal stem cells.

patients met the ACR70 criteria, and the DAS 28 score was less than 2.6. The levels of complement $\mathrm{C} 3$ and $\mathrm{C} 4$, routine blood tests, liver and kidney function, and serum immunoglobulin levels showed no significant changes before and after treatment. No serious adverse effects occurred during the treatment period, and so UC-MSCs were considered to exhibit immune regulation, prevent the release of inflammatory mediators, reduce soft tissue injury and play a role in other important effects that could alleviate clinical RA symptoms. Thus, safety has 
been demonstrated for the clinical treatment of RA. Additionally, we conducted prospective phase I/II studies in 10 juvenile idiopathic arthritis (JIA) children ${ }^{56}$ less than 17 years of age and 64 RA patients ${ }^{7}$ aged 18 to 64 years. The patients were treated with a $40 \mathrm{~mL}$ of a UC-MSC suspension product $\left(2 \times 10^{7}\right.$ cells $\left./ 20 \mathrm{~mL}\right)$ via intravenous injection. The ESR, $\mathrm{C}$ reactive protein (CRP) levels, and rheumatoid factor (RF) levels at 1 year and 3 years after treatment and anti-CCP at 3 years after treatment were significantly lower than those pretreatment $(\mathrm{P}<0.05)$. One year and three years after treatment, The HAQ and DAS28 decreased in comparison to the pretreatment levels $(\mathrm{P}<0.05)$. Overall, UC-MSC therapy can be a safe, effective, and feasible therapeutic option for RA patients. Liu and colleague showed that UC-MSCs have therapeutic potential in the treatment of RA. The results showed that UC-MSCs suppressed the various inflammatory effects of FLSs and $\mathrm{T}$ cells. In addition, the immunosuppressive activity of UC-MSCs could be prolonged by the participation of Tregs.

\section{Challenges and Perspectives}

Umbilical cord blood and tissue are ideal sources of MSCs because they can be obtained by noninvasive means more easily than from bone marrow. ${ }^{60}$ These cells have a lower relative risk of viral contamination and can be cryopreserved for autologous transplantation. However, the success rate of cultured MSCs from umbilical cord blood is not high, with one study suggesting only $6 \%$, while the success rate of cultured MSCs from umbilical cord blood can reach $100 \%$. Therefore, in recent years, the umbilical cord has become an important source of MSCs. Although the physiological characteristics and mechanisms of action of MSCs have been deeply studied, there are still many challenges to be solved in the treatment of RA. At present, there is no unified plan for stem cell transplantation to treat this disease, such as pretransplantation medication, transplanted cell numbers, and the optimal timing of treatments. The safety of the treatment and the suitable population still need long-term follow-up.

Genetic stability is affected by donors, tissue sources, and culture conditions during amplification, storage conditions, and the time of passage. ${ }^{62,63}$ Stultz et al, ${ }^{64}$ examined the chromosomal stability of BM-MSCs from different donors and algebraically analyzed the results. The results showed that there were more abnormal karyotypes in the third generation than in the 5th and 7th generations, and the abnormal karyotypes gradually decreased with increasing generations through genetic modeling of inheritance. According to different donor ages in the elderly and young groups, the algebraic model showed that donors from the elderly group were characterized mainly by chromosome translocation, while donors from the young group were characterized by aneuploidy, but in both the elderly group and the young group, there were no significant differences in chromosomes; chromosomal abnormality occurred mainly in the early stages of the algebraic model, and with more passage times, there were more mature and stable the chromosomes. Duarte and colleagues ${ }^{65}$ compared pre- and post cryopreservation of the human umbilical vein to assess the influence of chromosome status on MSCs and found that nonclone chromosome aberration was detected after frozen storage in liquid nitrogen, and the three short arms of a chromosome were inverted when the cells were stored for two months.

At present, the MSCs used to study regeneration technology mainly include BM-MSCs and UC-MSCs. ${ }^{7,10,50}$ Cord blood is from the umbilical cord near the fetal side of the placenta. This blood is rich in stem cells and progenitor cells and has the potential for multidirectional differentiation. There is no ethical problem in treatments with cord blood stem cells. China is a populous country with abundant availability of umbilical cord blood. Due to the lack of understanding and a scientific research platform, the development of umbilical cord blood has been neglected. BM-MSCs have more sufficient sources and are more convenient to collect than other types of MSCs and do not express MHC II molecules, so they cannot identify various antigenic mechanisms. Due to a lack of immune activation in two common stimulatory signal transduction pathways and immune tolerance, the incidence of GVHD is low. ${ }^{66}$ There are also primitive cells, such as endothelial progenitor cells, in umbilical cord blood, which are beneficial to vascular reconstruction and to improving blood supply at the site of injuries, as such cells play an auxiliary role in repair. Compared with BM-MSCs, UC-MSCs are more primitive and have stronger amplification abilities. The application of UC-MSCs does not involve ethical issues, and obtaining umbilical cord blood from newborns does not cause any harm to newborns. Because umbilical cord blood is protected by the placental barrier, it is less likely to cause viral infections during transplantation than bone marrow transplantation. Moreover, cord blood stem cells are extremely pure. Cord blood is easy to obtain and is an available resource. UC-MSC therapy involves no 
ethical issues and no tissue antigenicity and has a simple transplantation route, obvious and reliable efficacy, and no adverse side effects. UC-MSCs can promote the directional activation of stem cells and accelerate nerve function repair.

A large number of studies have confirmed that UCMSCs play a role in RA disease, but their mechanism remains unclear. There are challenges in the use of UCMSC therapy in clinical trials. For example, a multiple center randomized controlled trial (RCT) needs to be performed; a large sample size and clinical trials need to be constructed to verify the therapeutic effect; there is no unified standard for the optimal dose, optimal time, treatment frequency, approach, treatment conditions and indications of UC-MSC therapy for RA; and whether there will be other potential side effects is a problem that needs to be observed and solved in future research. In summary, UC-MSCs are studied not only in the laboratory but also in the clinic. All studies showed excellent efficacy, especially those utilizing UC-MSCs, which are a potential feasible method of RA treatment.

\section{Conclusion}

UC-MSCs show good efficacy and tolerability in RA patients and have emerged as a promising alternative in the management of RA. The pathogenesis of RA is related to disorders of immune mechanisms and cytokines. UCMSCs are widely available and have low immunogenic responses, and the limitations, such as the lack of traditional stem cell sources, allogeneic rejection, and ethics, have been overcome. As UC-MSCs have achieved a certain efficacy and few side effects in clinical practice, they are worthy of further large-sample RCTs for future evaluation.

\section{Abbreviations}

BM, bone marrow; BM-MSCs, bone marrow mesenchymal stem cells; RA, rheumatoid arthritis; UC-MSCs, Umbilical cord mesenchymal stem cells; DAS 28, Disease Activity Score 28-joint; AE, adverse events; ESR, erythrocyte sedimentation rate; RF rheumatoid factor; DMARDs, Drug Rheumatoid Arthritis With Disease-Modifying Drugs; SAE, Serious Adverse Events; DAS28-CRP, disease activity score 28-joint count using $\mathrm{C}$ reactive protein; HAQ-DI, health assessment questionnaire disability index; SDAI, Simplified Disease Activity Index; anti-CCP, anti-cyclic citrullinated peptide; HGB, hemoglobin; CIA, collagen-induced arthritis;
FLSs, fibroblast-like synoviocytes; FVC, forced vital capacity; ACR, American College of Rheumatology.

\section{Funding}

This research was supported by the Guangxi Zhuang Autonomous Region self-funded project (NO: Z20190359) and the Project of Liuzhou Science and Technology Bureau (No: 2018BJ10502).

\section{Disclosure}

The authors report no conflicts of interest in this work.

\section{References}

1. Otón T, Carmona L. The epidemiology of established rheumatoid arthritis. Best Pract Res Clin Rheumatol. 2019;33(5):101477. doi:10.1016/j.berh.2019.101477

2. Goldring SR. Pathogenesis of bone erosions in rheumatoid arthritis. Curr Opin Rheumatol. 2002;14(4):406-410. doi:10.1097/00002281200207000-00013

3. Subesinghe S, Bechman K, Rutherford AI, Goldblatt D, Galloway JB. A systematic review and metaanalysis of antirheumatic drugs and vaccine immunogenicity in rheumatoid arthritis. J Rheumatol. 2018;45(6):733-744. doi:10.3899/jrheum.170710

4. El-Gabalawy H. The preclinical stages of RA: lessons from human studies and animal models. Best Pract Res Clin Rheumatol. 2009;23 (1):49-58. doi:10.1016/j.berh.2008.11.004

5. Chang NC. Autophagy and stem cells: self-eating for self-renewal. Front Cell Develop Biol. 2020;8:138. doi:10.3389/fcell.2020.00138

6. Ross CL, Ang DC, Almeida-Porada G. Targeting mesenchymal stromal cells/pericytes (MSCs) with Pulsed Electromagnetic Field (PEMF) has the potential to treat rheumatoid arthritis. Front Immunol. 2019;10:266. doi:10.3389/fimmu.2019.00266

7. Wang L, Huang S, Li S, et al. Efficacy and safety of umbilical cord mesenchymal stem cell therapy for rheumatoid arthritis patients: a prospective phase $\mathrm{I} / \mathrm{Ii}$ study. Drug Des Devel Ther. 2019;13:4331-4340. doi:10.2147/DDDT.S225613

8. Wang L, Wang L, Cong X, et al. Human umbilical cord mesenchymal stem cell therapy for patients with active rheumatoid arthritis: safety and efficacy. Stem Cells Dev. 2013;22(23):3192-3202.

9. Lopez-Santalla M, Fernandez-Perez R, Garin MI. Mesenchymal stem/stromal cells for rheumatoid arthritis treatment: an update on clinical applications. Cells. 2020;9(8):1852. doi:10.3390/ cells9081852

10. Coelho A, Alvites RD, Branquinho MV, Guerreiro SG, Maurício AC. Mesenchymal stem cells (MSCs) as a potential therapeutic strategy in COVID-19 patients: literature research. Front Cell Develop Biol. 2020;8:602647. doi:10.3389/fcell.2020.602647

11. Wang L, Huang S, Dang Y, et al. Cord blood-derived cytokine-induced killer cellular therapy plus radiation therapy for esophageal cancer: a case report. Medicine. 2014;93(28):e340. doi:10.1097/MD.0000000000000340

12. Wienecke T, Gøtzsche PCJ. Paracetamol versus nonsteroidal antiinflammatory drugs for rheumatoid arthritis. Cochrane Database Syst Rev. 2004;(1):CD003789. doi:10.1002/14651858.CD003789. pub2.

13. Tiano HF, Loftin CD, Akunda J, et al. Deficiency of either cyclooxygenase (COX)-1 or COX-2 alters epidermal differentiation and reduces mouse skin tumorigenesis. Cancer Res. 2002;62 (12):3395-3401. 
14. Lovell DJ, Ruperto N, Goodman S, et al. Adalimumab with or without methotrexate in juvenile rheumatoid arthritis. $N$ Engl $\mathrm{J} \mathrm{Med}$. 2008;359(8):810-820.

15. Schett G, Emery P, Tanaka Y, et al. Tapering biologic and conventional DMARD therapy in rheumatoid arthritis: current evidence and future directions. Ann Rheum Dis. 2016;75(8):1428-1437.

16. Castillo J, Milani C, Mendez-Allwood DJE. Ofatumumab, a second-generation anti-CD20 monoclonal antibody, for the treatment of lymphoproliferative and autoimmune disorders. Expert Opin Investig Drugs. 2009;18(4):491-500.

17. Song S-NJ, Iwahashi M, Tomosugi N, et al. Comparative evaluation of the effects of treatment with tocilizumab and TNF- $\alpha$ inhibitors on serum hepcidin, anemia response and disease activity in rheumatoid arthritis patients. Arthritis Res Ther. 2013;15(5):1-10.

18. Downey CJ. Serious infection during etanercept, infliximab and adalimumab therapy for rheumatoid arthritis: a literature review. $J$ Rheum Dis. 2016;19(6):536-550.

19. Smolen JS, Van Der Heijde DM, St. Clair EW, et al. Predictors of joint damage in patients with early rheumatoid arthritis treated with high-dose methotrexate with or without concomitant infliximab: results from the ASPIRE trial. Arthritis Rheum. 2006;54 (3):702-710.

20. St. Clair EW, Van Der Heijde DM, Smolen JS, et al. Combination of infliximab and methotrexate therapy for early rheumatoid arthritis: a randomized, controlled trial. Arthritis Rheum2004;50(11):3432-3443.

21. Bartelds GM, Wijbrandts CA, Nurmohamed MT, et al. Clinical response to adalimumab: relationship to anti-adalimumab antibodies and serum adalimumab concentrations in rheumatoid arthritis. Ann Rheum Dis. 2007;66(7):921-926.

22. Raaschou P, Simard JF, Holmqvist M, Askling J. Rheumatoid arthritis, anti-tumour necrosis factor therapy, and risk of malignant melanoma: nationwide population based prospective cohort study from Sweden. BMJ. 2013;346(f1939).

23. Vizoso FJ, Eiro N, Costa L, et al. Mesenchymal stem cells in homeostasis and systemic diseases: hypothesis, evidences, and therapeutic opportunities. Int J Mol Sci. 2019;20(15):3738. doi:10.3390/ ijms20153738

24. Chen L, Qu J, Xiang C. The multi-functional roles of menstrual blood-derived stem cells in regenerative medicine. Stem Cell Res Ther. 2019;10(1). doi:10.1186/s13287-018-1105-9

25. Li Y, Jin D, Xie W, et al. Mesenchymal stem cells-derived exosomes: a possible therapeutic strategy for osteoporosis. Curr Stem Cell Res Ther. 2018;13(5):362-368. doi:10.2174/1574888X13666180403163456

26. Qu J, Zhang H. Roles of mesenchymal stem cells in spinal cord injury. Stem Cells Int. 2017;2017:5251313. doi:10.1155/2017/ 5251313

27. Zhou T, Li H-Y, Liao C, Lin W, Lin S. Clinical efficacy and safety of mesenchymal stem cells for systemic lupus erythematosus. Stem Cells Int. 2020;2020:6518508. doi:10.1155/2020/6518508

28. Ferro F, Elefante E, Luciano N, Talarico R, Todoerti M. One year in review 2017: novelties in the treatment of rheumatoid arthritis. Clin Exp Rheumatol. 2017;35(5):721-734.

29. Silvagni E, Di Battista M, Bonifacio AF, Zucchi D, Governato G, Scirè CA. One year in review 2019: novelties in the treatment of rheumatoid arthritis. Clin Exp Rheumatol. 2019;37(4):519-534.

30. Cheung TT, McInnes IB. Future therapeutic targets in rheumatoid arthritis? Semin Immunopathol. 2017;39(4):487-500. doi:10.1007/ s00281-017-0623-3

31. Luo $\mathrm{Y}, \mathrm{Wu} \mathrm{W}, \mathrm{Gu} \mathrm{J}$, et al. Human gingival tissue-derived MSC suppress osteoclastogenesis and bone erosion via CD39-adenosine signal pathway in autoimmune arthritis. EBioMedicine. 2019;43:620-631. doi:10.1016/j.ebiom.2019.04.058

32. Luque-Campos N, Contreras-López RA, Jose Paredes-Martínez M, et al. Mesenchymal stem cells improve rheumatoid arthritis progression by controlling memory $\mathrm{T}$ cell response. Front Immunol. 2019;10:798. doi:10.3389/fimmu.2019.00798
33. Liu R, Zhao P, Tan W, Zhang M. Cell therapies for refractory rheumatoid arthritis. Clin Exp Rheumatol. 2018;36(5):911-919.

34. Haikal SM, Abdeltawab NF, Rashed LA, Abd El-Galil TI, Elmalt HA, Amin MA. Combination therapy of mesenchymal stromal cells and interleukin-4 attenuates rheumatoid arthritis in a collagen-induced murine model. Cells. 2019;8(8):823. doi:10.3390/ cells 8080823

35. Li E, Zhang Z, Jiang B, Yan L, Park JW, Xu RH. Generation of mesenchymal stem cells from human embryonic stem cells in a complete serum-free condition. Int $J$ Biol Sci. 2018;14 (13):1901-1909. doi:10.7150/ijbs.25306

36. Hynes K, Bright R, Proudman S, Haynes D, Gronthos S, Bartold M. Immunomodulatory properties of mesenchymal stem cell in experimental arthritis in rat and mouse models: a systematic review. Semin Arthritis Rheum. 2016;46(1):1-19. doi:10.1016/j. semarthrit.2016.02.008

37. Andrzejewska A, Lukomska B, Janowski M. Concise review: mesenchymal stem cells: from roots to boost. Stem Cells. 2019;37 (7):855-864. doi:10.1002/stem.3016

38. Liu Y, Mu R, Wang S, et al. Therapeutic potential of human umbilical cord mesenchymal stem cells in the treatment of rheumatoid arthritis. Arthritis Res Ther. 2010;12(6):R210. doi:10.1186/ar3187

39. Berglund AK, Fisher MB, Cameron KA, Poole EJ, Schnabel LV. Transforming growth factor- $\beta 2$ downregulates Major Histocompatibility Complex (MHC) I and MHC II surface expression on equine bone marrow-derived mesenchymal stem cells without altering other phenotypic cell surface markers. Front Vet Sci. 2017;4:84. doi:10.3389/fvets.2017.00084

40. Karahuseyinoglu S, Cinar O, Kilic E, et al. Biology of stem cells in human umbilical cord stroma: in situ and in vitro surveys. Stem Cells. 2007;25(2):319-331. doi:10.1634/stemcells.2006-0286

41. Wang Q, Li X, Luo J, et al. The allogeneic umbilical cord mesenchymal stem cells regulate the function of $\mathrm{T}$ helper 17 cells from patients with rheumatoid arthritis in an in vitro co-culture system. BMC Musculoskelet Disord. 2012;13(1):249. doi:10.1186/14712474-13-249

42. Gu J, Lin CM, Gu W, et al. [Immunomodulatory effect of UC-MSC on function of immunocytes of rats with collagen type II induced arthritis]. Zhongguo Shi Yan Xue Ye Xue Za Zhi. 2014;22(1):166-170. [Chinese]

43. Zhao Q, Ren H, Han Z. Mesenchymal stem cells: immunomodulatory capability and clinical potential in immune diseases. $J$ Cell Immunother. 2016;2(1):3-20. doi:10.1016/j.jocit.2014.12.001

44. Shou K, Huang Y, Qi B, et al. Induction of mesenchymal stem cell differentiation in the absence of soluble inducer for cutaneous wound regeneration by a chitin nanofiber-based hydrogel. J Tissue Eng Regen Med. 2018;12(2):e867-e880. doi:10.1002/term.2400

45. Fu X, Liu G, Halim A, Ju Y, Luo Q, Song AG. Mesenchymal stem cell migration and tissue repair. Cells. 2019;8(8):784.

46. Chen L, Carlton M, Chen X, et al. Effect of fibronectin, FGF-2, and BMP4 in the stemness maintenance of BMSCs and the metabolic and proteomic cues involved. Stem Cell Res Ther. 2021;12(1):165. doi:10.1186/s13287-021-02227-7

47. Xu L, Liu Y, Sun Y, et al. Tissue source determines the differentiation potentials of mesenchymal stem cells: a comparative study of human mesenchymal stem cells from bone marrow and adipose tissue. Stem Cell Res Ther. 2017;8(1):275. doi:10.1186/s13287-017-0716-x

48. Zhang B, Luo Q, Halim A, Ju Y, Morita Y, Song G. Directed differentiation and paracrine mechanisms of mesenchymal stem cells: potential implications for tendon repair and regeneration. Curr Stem Cell Res Ther. 2017;12(6):447-454. doi:10.2174/ 1574888X12666170502102423

49. Song H, Yin Z, Wu T, et al. Enhanced effect of tendon stem/progenitor cells combined with tendon-derived decellularized extracellular matrix on tendon regeneration. Cell Transplant. 2018;27 (11):1634-1643. doi:10.1177/0963689718805383 
50. Wu Y, Wang J, Scott PG, Tredget EE. Bone marrow-derived stem cells in wound healing: a review. Wound Repair Regen. 2007;15 (Suppl s1):S18-26. doi:10.1111/j.1524-475X.2007.00221.x

51. Herdrich BJ, Lind RC, Liechty KW. Multipotent adult progenitor cells: their role in wound healing and the treatment of dermal wounds. Cytotherapy. 2008;10(6):543-550. doi:10.1080/ 14653240802345820

52. Cha J, Falanga V. Stem cells in cutaneous wound healing. Clin Dermatol. 2007;25(1):73-78. doi:10.1016/j.clindermatol.2006.10.002

53. Fu X, Li H. Mesenchymal stem cells and skin wound repair and regeneration: possibilities and questions. Cell Tissue Res. 2009;335 (2):317-321. doi:10.1007/s00441-008-0724-3

54. Suh W, Kim KL, Kim JM, et al. Transplantation of endothelial progenitor cells accelerates dermal wound healing with increased recruitment of monocytes/macrophages and neovascularization. Stem Cells. 2005;23(10):1571-1578. doi:10.1634/stemcells.20040340

55. Volk SW, Radu A, Zhang L, Liechty KW. Stromal progenitor cell therapy corrects the wound-healing defect in the ischemic rabbit ear model of chronic wound repair. Wound Repair Regen. 2007;15 (5):736-747. doi:10.1111/j.1524-475X.2007.00277.x

56. Wei Z, Yuan J, Wang G, Ocansey DKW, Xu Z, Mao FJSCI. Regulatory effect of mesenchymal stem cells on $t$ cell phenotypes in autoimmune diseases. Stem Cell Int. 2021;2021:5583994. doi: $10.1155 / 2021 / 5583994$

57. Mattar P, Bieback K. Comparing the immunomodulatory properties of bone marrow, adipose tissue, and birth-associated tissue mesenchymal stromal cells. Front Immunol. 2015;6:560.

58. Song J-Y, Kang HJ, Hong JS, et al. Umbilical cord-derived mesenchymal stem cell extracts reduce colitis in mice by re-polarizing intestinal macrophages. Sci Rep. 2017;7(1):1-11.
59. Greish S, Abogresha N, Abdel-Hady Z, Zakaria E, Ghaly M, Hefny M. Human umbilical cord mesenchymal stem cells as treatment of adjuvant rheumatoid arthritis in a rat model. World J Stem Cells. 2012;4(10):101-109. doi:10.4252/wjsc.v4.i10.101

60. Park EH, Lim HS, Lee S, et al. Intravenous infusion of umbilical cord blood-derived mesenchymal stem cells in rheumatoid arthritis: a phase Ia clinical trial. Stem Cells Transl Med. 2018;7(9):636-642. doi:10.1002/sctm.18-0031

61. Qi T, Gao H, Dang Y, Huang S, Peng M. Cervus and cucumis peptides combined umbilical cord mesenchymal stem cells therapy for rheumatoid arthritis. Medicine. 2020;99(28):e21222. doi:10.1097/ MD.0000000000021222

62. Augello A, Tasso R, Negrini SM, Cancedda R, Pennesi G. Cell therapy using allogeneic bone marrow mesenchymal stem cells prevents tissue damage in collagen-induced arthritis. Arthritis Rheum. 2007;56(4):1175-1186. doi:10.1002/art.22511

63. Sensebé L, Tarte K, Galipeau J, et al. Limited acquisition of chromosomal aberrations in human adult mesenchymal stromal cells. Cell Stem Cell. 2012;10(1):9-10. doi:10.1016/j.stem.2011.12.005

64. Stultz BG, McGinnis K, Thompson EE, Lo Surdo JL, Bauer SR, Hursh DA. Chromosomal stability of mesenchymal stromal cells during in vitro culture. Cytotherapy. 2016;18(3):336-343. doi:10.1016/j.jcyt.2015.11.017

65. Duarte DM, Cornélio DA, Corado C, et al. Chromosomal characterization of cryopreserved mesenchymal stem cells from the human subendothelium umbilical cord vein. Regen Med. 2012;7(2):147-157. doi:10.2217/rme.11.113

66. Zheng G, Liu Y, Jing Q, Zhang L. Differentiation of human umbilical cord-derived mesenchymal stem cells into hepatocytes in vitro. Biomed Mater Eng. 2015;25(1 Suppl):145-157. doi:10.3233/BME141249

\section{Publish your work in this journal}

Drug Design, Development and Therapy is an international, peerreviewed open-access journal that spans the spectrum of drug design and development through to clinical applications. Clinical outcomes, patient safety, and programs for the development and effective, safe, and sustained use of medicines are a feature of the journal, which has also been accepted for indexing on PubMed Central. The manuscript management system is completely online and includes a very quick and fair peer-review system, which is all easy to use. Visit http://www. dovepress.com/testimonials.php to read real quotes from published authors. 